\title{
The role of EDEM3, Derlin-1 and Derlin-2 proteins in the ricin P250A cytotoxicity and its retrotranslocation from the endoplasmic reticulum to the cytosol
}

\author{
Hanna Sominka, Monika Słomińska-Wojewódzka \\ Department of Medical Biology and Genetics, Faculty of Biology, University of Gdańsk, Wita Stwosza 59 Street, \\ 80-308 Gdańsk, Poland \\ Corresponding author: hanna.sominka@phdstud.ug.edu.pl \\ DOI: $10.31708 /$ spi1.2018.2
}

\section{Introduction}

ERAD-ER-associated degradation is a part of a protein quality control system operating in the endoplasmic reticulum (ER), which has an impact on process determining the proper functioning of all eukaryotic cells. Many of all newly synthesized proteins are produced in the ER. Some of them may fail to attain their native structure and have to be transported to the cytosol for proteasomal degradation (ERAD). The major group of chaperones which recognize terminally misfolded proteins is EDEM family (EDEM1, EDEM2, EDEM3) (Słomińska-Wojewódzka et al., 2006). The second type of proteins important in ERAD are Derlin family: Derlin-1, Derlin-2, and Derlin-3 which are thought to form a retrotranslocation channel (Oda et al., 2016). Ricin is a protein toxin that utilizes the ERAD pathway in its transport from the ER to the cytosol where it acts. It is heterodimeric holotoxin composed of an A-chain (RTA) connected to a cell binding lectin B-chain (RTB). RTA contains hydrophobic $\mathrm{C}$-terminal region. Substitution of proline into alanine in position 250 (P250A) of this region alters the secondary structure of ricin and decreases its cytotoxicity (Słomińska-Wojewódzka \& Sandvig, 2013).

\section{Materials and Methods}

Overexpression of EDEM3, Derlin-1, and Derlin-2 was obtained by HEK293 cells transfection using appropriate cDNA constructs. Studies on the ricin P250A cytotoxicity were performed on cells which were incubated with different concentrations of ricin P250A. Retrotranslocation of ricin to the cytosol examined by the permeabilization of cell membrane using detergentdigitonin allows separating the cytosolic fraction from membranes. Amount of RTA transported to the cytosol was calculated.

\section{Results}

The cytotoxicity and permeabilization experiments indicated that high expression of EDEM3 sensitizes HEK293 cells against ricin P250A and promotes retro- translocation of the modified toxin to the cytosol. The same effect was observed in the case of Derlin-1 overproduction. High level of Derlin-2 does not influence cytotoxicity RTA P250A in HEK293 cells.

\section{Discussion}

Due to its high toxicity, ricin is considered as a biological weapon. On the other hand, detailed knowledge of the ricin intercellular transport mechanisms raises the possibility of using it in medicine as a component of modern immunotoxins and vaccines (SłomińskaWojewódzka \& Sandvig, 2013). The previous study reported that EDEM1 and EDEM2 are involved in ricin transport out of the ER (Sokołowska et al., 2011). In contrast to wild-type RTA, P250A transport to the cytosol appears to be EDEM1- and EDEM2- independent. Moreover, RTA P250A do not interact with EDEM1 and EDEM2 (Olivari \& Molinari, 2007; SłomińskaWojewódzka et al., 2014). The results of obtained experiments, in which studied cytotoxicity and transported RTA P250A from the ER to the cytosol showed that EDEM3 overexpression promotes its retrotranslocation and sensitizes HEK293 cells against modified ricin. Another family of proteins which are important in this process is Derlin family. Derlin-2 is a protein that exhibits near $30 \%$ sequence identity with Derlin-1 and possesses four transmembrane regions of similar topology to Derlin-1. Previous studies indicated that Derlin-1 and Derlin-2 might play a role in the dislocation and degradation of misfolded proteins from the ER (Lilley \& Ploegh, 2005). In the present study of the role of Derlin-1 and Derlin-2 in RTA P250A transport from the endoplasmic reticulum to the cytosol, it was observed that Derlin-1 promotes toxin retrotranslocation and cause an increase of its cytotoxicity almost two-fold. High expression of Derlin-2 protein does not affect the modified ricin transport. In conclusion, these results contribute to the general understanding of ricin intracellular transport, the role of the hydrophobic region of RTA in ricin 
cytotoxicity and the mechanisms of the recognition of misfolded proteins in the ER.

\section{References}

Lilley B.N, Ploegh H.L., (2005). Multiprotein complexes that link dislocation, ubiquitination, and extraction of misfolded proteins from the endoplasmic reticulum. PNAS, 102: 14296-14301

Słomińska-Wojewódzka M., Gregers T. F., Wälchli S., Sandvig K., (2006). EDEM is involved in ricin retrotranslocation of ricin from the endoplasmic reticulum to the cytosol. Mol Biol Cell, 17: 16641675

Słomińska-Wojewódzka M., Sandvig K., (2013). Ricin and Ricin-Containing Immunotoxins: Insights into Intracellular Transport and Mechanism of action in Vitro; Antibodies, 2, 236-269

Słomińska-Wojewódzka, M., Pawlik, A., Sokołowska, I., Antoniewicz, J., Węgrzyn, G., Sandvig, K., (2014). The role of EDEM2 compared with EDEM1 in ricin transport from the endoplasmic reticulum to the cytosol. Biochem. J. 457, 485-496

Sokołowska I., Węgrzyn G., Słomińska-Wojewódzka, M., Sandvig, K., (2011). A single point mutation in ricin Achain increases toxin degradation and inhibits EDEM1dependent ER retrotranslocation; Biochem.J. 436,371385

Oda Y., Okada T., Yoshida H., Kauffman R. J., Nagata K., Mori K., (2006). Derlin-2 and Derlin-3 are regulated by the mammalian unfolded protein response and are required for ER-associated degradation. J Biol Chem, 172: $383-393$

Olivari S., Molinari M., (2007). Glycoprotein folding and the role of EDEM1, EDEM2 and EDEM3 in degradation of folding-defective glycoproteins. FEBS Lett, 581: 36583664 